Revista Perspectivas Online: Exatas \& Engenharias Dezembro de 2017, Vol.7, n 19 , p. 20-35 ISSN: 2236-885X (Online)

DOI: $10.25242 / 885 \times 7192017738$

\title{
PROPRIEDADES ELETRÔNICAS DA LIGA QUASICRISTALINA AICuFe
}

\author{
Luciano Nascimento1*
}

\section{RESUMO}

NASCIMENTO, L. Propriedades Eletrônicas da Liga Quasicristalina AlCuFe. Perspectivas Online: Exatas \& Engenharias, v. 7, n.19, p.20-35,2017.

Este trabalho tem como objetivo principal mostrar um estudo sobre o comportamento da liga quasicristalina $\mathrm{AlCuFe}$ com relação às suas propriedades estruturais e eletrônicas. Com relação às propriedades estruturais foi dado ênfase aos padrões de difração de raios X e por evolução microestrutural pela a microscopia eletrônica de varredura. $\mathrm{O}$ estudo das propriedades eletrônicas seguiu a seqüência de Fibonacci para determinar os espectros eletrônicos da liga estudada. Foi mostrado que as propriedades eletrônicas destas, representam sistemas intermediários entre ordem e desordem, em relação sequência de Fibonacci que descreve de forma geral o calor específico dos quasicristais. Destacamos que seu comportamento está ligado diretamente com o calor específico, onde podemos usar o formalismo da mecânica estatística, que descreve as pequenas oscilações de energia térmica em regiões de baixas temperaturas, pois estas estruturas quasiperiódicas quasicristalinas obedecem à sequência de Fibonacci generalizada ao obter o número de banda de energia do espectro fractal no conjunto triádico de Cantor pela a sequência de Fibonacci.

Palavras-chave: Espectros Eletrônicos; Calor Específico; Sequência de Fibonacci; Quasicristal AlCuFe. 


\begin{abstract}
This work aims to show a study on the behavior of quasicristalina league $\mathrm{AlCuFe}$ with respect to their structural and electronic properties. With respect to structural properties was stressed to the standards of X-Ray Diffraction and microstructural evolution by the Scanning Electron Microscopy. The study of electronic properties followed the Fibonacci sequence to determine the electronic spectra of the alloy studied. It was shown that the electronic properties of these represent intermediate systems between order and

disorder, compared Fibonacci sequence that generally describes the specific heat of quasicrystals. We emphasize that their behavior is linked directly to the specific heat, where we can use the formalism of statistical mechanics, which describes small thermal power surges in low temperature regions, because these structures quasiperiodic quasicrystalline obey the generalized Fibonacci sequence to get the number of energy band fractal spectrum triadic Cantor ensemble by the Fibonacci sequence.
\end{abstract}

Key-words: Electronic Spectra; Specific Heat; Fibonacci Sequence; Quasicrystal AlCuFe.

${ }^{1}$ Universidade Federal da Paraíba (UFPB) - Departamento de Física (DF/CCEN) - Cidade Universitária, Caixa Postal: 5008, João Pessoa, PB, CEP: 58059-900, Brasil.

(*)e-mail: luciano.ufpe@gmail.com

Data de chegada: 11/07/2015 Aceito para publicação: 21/11/2017

Persp. Online: exatas \& eng., Campos dos Goytacazes, 19 (07) 20-35 - 2017 


\section{INTRODUÇÃO}

Os quasicristais são estruturas atômicas desordenadas que exibem ordem translacional quasiperiódicas de longo alcance, com simetria cristalograficamente proibida, são bons isolantes elétricos e térmicos além de apresentar alta dureza e resistência mecânica ao desgaste a corrosão [1]. Eles também se caracterizam por uma baixa condutividade térmica e alta resistividade. Os métodos de preparação destes materiais quasicristalinos são por solidificação rápida (melt-spinning, fundição de forno arco e atomização a gás, moagem de alta energia, eletrodeposição e evaporação de gás a laser, e etc.) [2].

Eles se constituem em uma classe intermediária entre os materiais amorfos e os cristalinos, quanto ao grau e característica de ordenamento, pois um quasicristal pode ser relacionado como constituído de aglomerados icosaédricos de átomos metálicos, todos orientados da mesma forma, e separados por quantidades variáveis de materiais desordenados, estas características dos quasicristais são bem distintas em relação às características dos metais convencionais [3]. Quasicristais são termodinamicamente estáveis e metaestáveis existem, na maioria, na forma de ligas ternárias (AlPdMn, AlCuFe, AlCuRu, etc.) e binárias (AlMn,AlPd, ZrPt, CoBe). Os quasicristais podem ser utilizados como aditivos e em revestimentos devido às suas propriedades de resistência e tribológicas, os quais não mudam a composição do objeto, mas significativamente aumentam a resistência ao desgaste, durabilidade quanto à corrosão, e a resistência mecânica de sua superfície [4].

A maioria destas ligas quasicristalinas é irreversível e transformam em cristais regulares quando do aquecimento a altas temperaturas. $\mathrm{O}$ interesse pelos quasicristais advém, principalmente, dessas suas propriedades físico-químicas paradoxais, para sistemas metálicos, e de suas propriedades eletrônicas, além, evidentemente, de suas características macroestrutural e catalítica [5]. Realmente, as ligas quasicristalinas mostram comportamentos fundamentalmente diferentes comparados às ligas metálicas cristalinas, com composições muito próximas, como reportado por quasicristais ricos em alumínio, por exemplo, tem condutividade elétrica muito baixa, quando comparada com ligas de base alumínio cristalinas ou amorfas a baixas temperaturas [6]. Além do mais, essa condutividade diminui com o aumento da temperatura, contrariando o comportamento dos metais comuns.

Em baixas temperaturas, as ligas quasicristalinas i-AlCuFe e i-AlPdMn têm propriedade térmica semelhante à de alguns óxidos, tais como os de zircônia, que são considerados excelentes isolantes, a parti de suas propriedades eletrônicas dessa liga sugerem que suas propriedades

Persp. Online: exatas \& eng., Campos dos Goytacazes, 19 (07) 20-35 - 2017

seer.perspectivasonline.com.br 
superficiais podem ser evidenciadas pelos padrões da maioria dos metais de transição [7]. A baixa condutividade elétrica dessa liga é atribuída, em parte, à supressão da densidade dos estados do nível de Fermi, o qual é chamado de "pseudogap". Convém salientar que os "gaps" (vazios, vácuos) são maiores em quasicristais do que em materiais cristalinos [8]. Este trabalho tem como objetivo estudar a sequência de Fibonacci generalizada para sistemas quasicristalino e a determinação do seu calor específico via o uso formalismo da mecânica estatística, descrever os processos métodos de obteção e a utilização desta liga quasicristalina $\mathrm{AlCuFe}$ em várias aplicações em função de suas propriedades eletrônicas de modo a facilitar o entendimento de seu comportamento físico.

\section{TEORIA}

\subsection{Propriedades eletrônicas em quasicristais}

Com a descoberta dos quasicristais de simetria icosaedral numa liga AlMn por Shechtman et al., (1984), levaram-se uma intensa pesquisa sobre a estrutura e propriedades eletrônicas destes novos materiais [9]. Estes materiais podem ser fabricados por moagem mecânica de alta energia, por solidificação rápida ou convencional, deposição física de vapor assistida por plasma (PAPVD).

Quasicristais têm muitas propriedades interessantes, como alta dureza, baixa condutividade elétrica e térmica, baixa energia de superfície, acompanhado por um baixo coeficiente de atrito, oxidação razoável, forte resistência à corrosão e propriedades ópticas incomuns que não são observadas em outras ligas cristalinas e ótimos armazenadores de hidrogênio [10]. O sistema de liga ternária $\mathrm{AlCuFe}$ possui uma fase icosaedral estável e várias fases aproximadas em pequeno range de concentração devidos aos efeitos eletrônicos observados na superfície de Fermi [11].

À temperatura ambiente exibem uma condutividade térmica muito baixa, atípico para ligas algumas ligas nanocristalinas. A sua resistividade elétrica é muito elevada e exibem um coeficiente de temperatura negativa, reminiscente de não-metais. Estas propriedades fazem com que estes sistemas quasicristalinos tenham certo número de diferentes áreas de aplicação como, a saber, em revestimentos resistentes ao desgaste mecânicos e corrosivos, e tendo ótimas propriedades catalíticas, devido a sua espessa camada de óxidos intermetálicos.

A estabilização de uma estrutura icosaédrica quasiperiodica é presumivelmente devido à estrutura eletrônica, em particular que resulta em um pseudogap na energia de Fermi, conhecido em compostos Hume-Rothery (HR), por apresentar elétrons de condução livres em sua superfície [12]. Este pseudogap também leva ao comportamento "antiaderente", interessante, por exemplo, em

Persp. Online: exatas \& eng., Campos dos Goytacazes, 19 (07) 20-35 - 2017 seer.perspectivasonline.com.br 
revestimento de superfícies em utensilios domésticos de cozinha. Dentro dos pseudogaps dos quasicristais, observamos estados eletrônicos supercríticos. Nos compostos de Hume-Rothery, a estrutura do cristal é estabilizada por uma interação eficaz entre a superfície Fermi e da zona de Brillouin-Jones, devido ao espalhamento de elétrons sobre os planos da rede. Isto resulta na redução da energia cinética total dos elétrons da banda de condução pela abertura de uma pseudogap ao nível de Fermi, aumentando a densidade da ocupada (ligação), e aumentando densidade dedesocupaçãodo (antiligação) nos níveis energia [13].

Tal mecanismo está previsto para ser ativo nos quasicristais icosaédricos devido ao fato de que a sua zona de pseudo-Brillouin está próximo de uma região esférica. Isto se torna o estudo de suas propriedades químicas e superficias,quando a sua estrutura eletrônica,possui efeitos termodinâmicos interessantes devido às transições de fases, qua causa uma composição de estruturas estáveis e metaestável entre as propriedades eletrônicas e a estabilidade de fase nestes materiais. Através das técnicas de caracterização de raios X e da microscopia de varredura eletrônica, a estrutura de ordem de longo alcance e as fases presentes no quasicristal, relacionando com a sua microestrutura icosaedral, estão ilustradas nas Figuras 1 e 2 abaixo [14]. Essas fases são utilizadas como pontos de partida para a análise estrutural do quasicristal icosaedral. Assim esperamos descrever os efeitos de transporte eletrônicos, catalíticos e estruturas eletrônicas entre estas fases. As ligas icosaedrais AlCuFe são de grande interesse comercial por apresentarem baixo coeficiente de atrito,elevada dureza e boa resistência a corrosão,pois estas ligas apresentam, ainda, baixo coeficiente de expansão térmica e elasticidade, caracterizada pelo Módulo de Young,bem próximas dos metais cristalinos. De todas as propriedades dos materiais quasicristalinos, a mais notável seja a baixa energia superficial e baixo coeficiente de atrito,pois elas estão relacionadas diretamente com as sua propriedade eletrônica,devido a energia de Fermi nos pesusdogaps. Os quasicristais é bastantemente utilizados como reforço em materiais compósitos ou mesmo para aplicação em revestimentos na forma de pó com garnulometria bastante fina.

Persp. Online: exatas \& eng., Campos dos Goytacazes, 19 (07) 20-35 - 2017 seer.perspectivasonline.com.br 


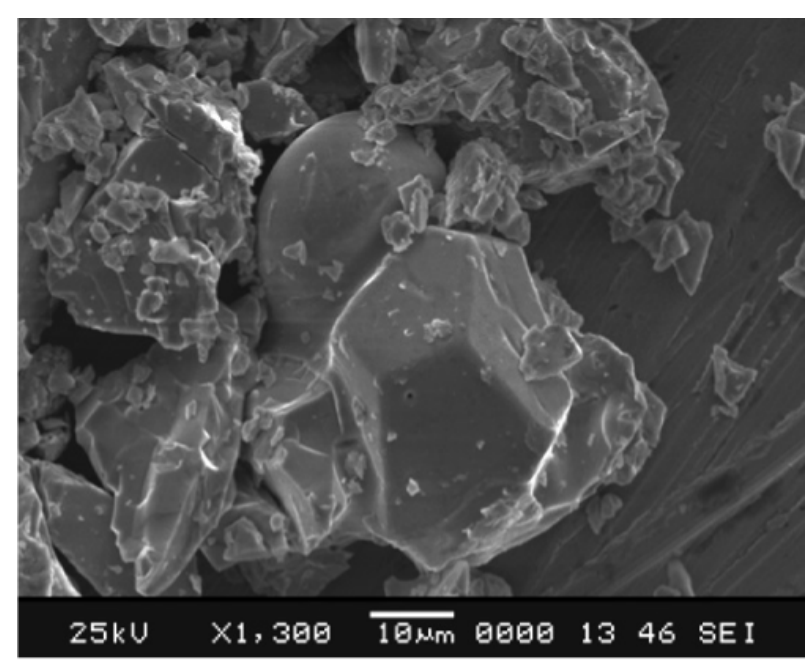

Figura 1 - MEV do quasicristal icosaedral AlCuFe.

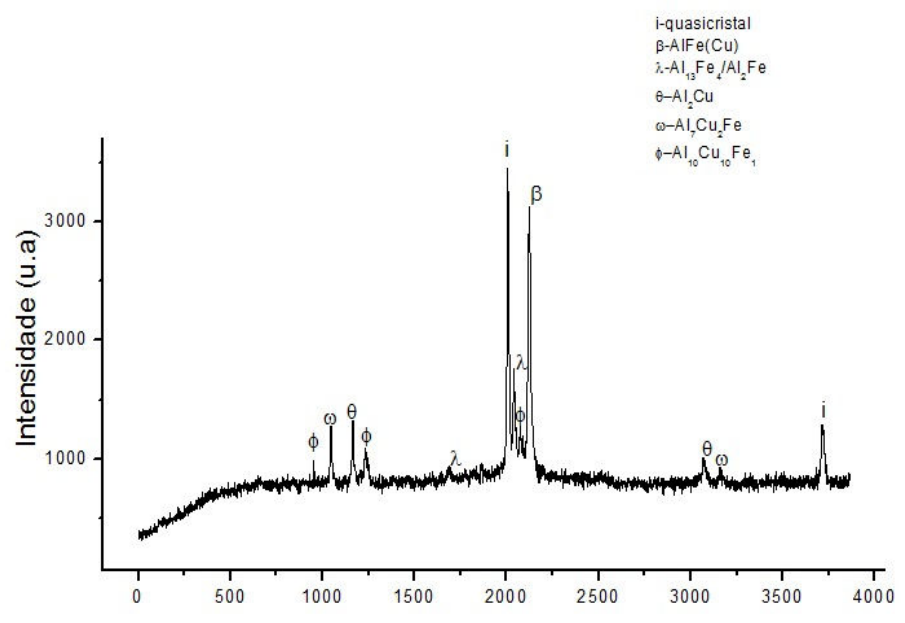

$(2 \theta)$

Figura 2 - Difratograma de raios $\mathrm{X}$ do quasicristal icosaedral AlCuFe.

\subsection{Sequência de Fibonacci Generalizada em sistemas quasiperiódicos}

A sequência de Fibonacci generalizada é uma relação de recorrência definida por,

$F_{n}=F_{n-1}+F_{n-2}$

, onde $n \geq 2$ e seus primeiros termos são $(1,1,2,3,5,8,13,21,34, \ldots)$. Daí afirmar que os números da sequência de Fibonacci são gerados adicionando os dois números antecessores da sequência, após especificar as condições iniciais. Esta sequência é infinita, e tem uma característica muito interessante:

Persp. Online: exatas \& eng., Campos dos Goytacazes, 19 (07) 20-35 - 2017

seer.perspectivasonline.com.br 
no limite $n \rightarrow \infty$, a razão entre dois termos consecutivos $F_{n} / F_{n-1}$ tende para um número irracional, que, para os matemáticos, é considerado o número irracional mais puro, e é comumente conhecido pelo nome de razão áurea ou número de ouro, a saber [15]:

$\tau=\frac{(1+\sqrt{5})}{2}=1,611803398874 \ldots$

Um fato curioso é que todos os termos da sequência de Fibonacci podem ser gerados a partir da razão áurea, através da relação:

$F_{n}=\frac{\tau^{n}-\left(-\frac{1}{\tau}\right)^{n}}{\sqrt{5}}$

ou seja, uma sequência de números inteiros é gerada por potências de números irracionais. Geralmente, a sequência de Fibonacci é usada para explicar e descrever a sistemas físicos, incluindo estudos de propriedades supercondutoras, sistemas de ligas amorfas e quasicristalinas, incluindo, plamon-polaritons em superredes do tipo Fibonacci constituídas de materiais dielétricos e transmissão de luz, com propriedades fotônica. Em estruturas quasiperiódicas do tipo amorfas e quasicristalinas, este tipo de sequência é construída segundo a sequência de Fibonacci generalizada que, pode ser obtida a partir de uma relação de recorrência, como discutido anteriormente, formando uma cadeia binária que pode ser crescida experimentalmente pela justaposição de dois blocos de construção A e B, de maneira que o n-ésimo estágio da sequência $S_{n}$ é gerado pela relação recursiva dada por,

$S_{n}=S_{n-1}^{p} S_{n-2}^{q},(n \geq 2)$

com $\mathrm{S}_{0}=\mathrm{B}$ e $\mathrm{S}_{1}=\mathrm{A}$. Os índices $\mathrm{p}$ e q são números inteiros positivos arbitrários e são eles que determinam o tipo de sequência a ser gerada. A quantidade $S_{n}^{p}\left(S_{n}^{q}\right)$ representa $\mathrm{p}$ (q) repetições adjacentes de Sn para uma rede quasiperiódicas em sistemas amorfo-quasicristalino, quando $\mathrm{p}=\mathrm{q}=$ 1,temos a sequência de Fibonacci do tipo normal. Uma maneira equivalente de gerar a sequência de Fibonacci generalizado é pela seguinte relação substitucional para estrutura com propriedades eletrônicas em sistemas quasiperiódicos, do tipo:

$B \rightarrow A, A \rightarrow A^{p} B^{q}$

Persp. Online: exatas \& eng., Campos dos Goytacazes, 19 (07) 20-35 - 2017 seer.perspectivasonline.com.br 
onde $\mathrm{A}^{\mathrm{p}}\left(\mathrm{B}^{\mathrm{q}}\right)$ é uma cadeia de $\mathrm{pA}$ 's ( $\mathrm{qB}$ 's). O número total de blocos $\mathrm{A}$ e $\mathrm{B}$ em $\mathrm{Sn}$ é igual ao número de Fibonacci generalizado $F_{n}(p ; q)$, que é dado pela relação de recorrência a seguir,

$F_{n}(p, q)=p F_{n-1}(p, q)+q F_{n-2}(p, q),(n \geq 2)$

O valor característico de $\sigma(p, q)$, que é a razão $F_{n}(p, q) / F_{n-1}(p, q)$ no limite é dado pela solução positiva da equação quadrática do tipo,

$\sigma^{2}-p \sigma-q=0$

ou podemos explicitar da seguinte forma

$\sigma(p, q)=\lim _{n \rightarrow \infty} \frac{\mathrm{F}_{\mathrm{n}}(\mathrm{p}, \mathrm{q})}{\mathrm{F}_{\mathrm{n}-1}(\mathrm{p}, \mathrm{q})}=\frac{p \pm \sqrt{p^{2}+4 q}}{2}$

Para os quasicristais e ligas amorfas, estes sistemas são formados segundo as sequências de Fibonacci generalizada, onde as flutuações de energia e agitações térmicas é mais pronunciadas de suas propriedades físicas (no nosso caso, o espectro de propagação de magnons em quasicristais e em ligas amorfas, como magnônicos do tipo Fibonacci em estruturas quasiperiódicas [16]. Portanto, a sequência de Fibonacci serve para determinar o espectro eletrônico de uma cadeia quasiperiódica em sistemas quasicristalinos.

\subsection{Espectros Eletrônicos de Quasicristais via Modelo de Bandas Contínuas}

As propriedades do calor específico em nível de propriedades eletrônicas associadas com aproximações hierárquicas sucessivas $(k=1,2,3, \ldots)$ relacionadas aos espectros de Cantor são construídas com seqüências de intervalos contínuos em espectros de energia em quasicristais [17]. Muitos modelos físicos sobre quasicristais, assim como os resultados experimentais relatados, exibem espectros de energia fractal. Certamente espectros do tipo Cantor triádico não correspondem a espectros reais, ou seja, eles não possuem contrapartida na natureza. Por outro lado, mesmo em se tratando de um típico fractal matemático, as informações obtidas pelo grupo do Tsallis para o calor

Persp. Online: exatas \& eng., Campos dos Goytacazes, 19 (07) 20-35 - 2017 seer.perspectivasonline.com.br 
específico foram extremamente interessantes. A Figura 3 abaixo ilustra a construção do espectro de energia do conjunto de Cantor em quasicristais.

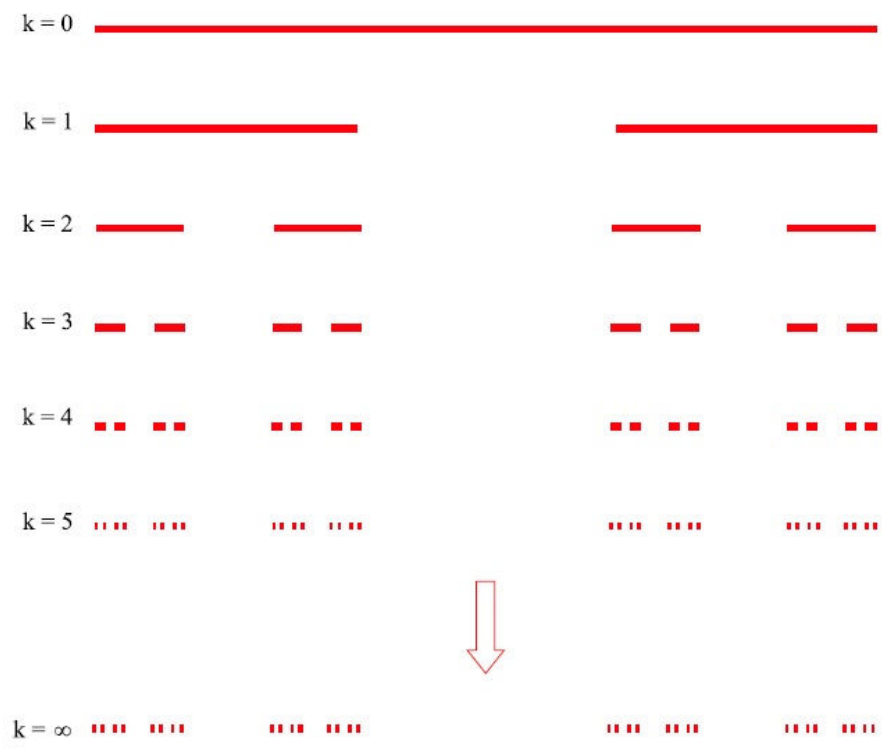

Figura 3 - A construção do conjunto de Cantor triádico no espectro de energia do quasicristal.

No limite, quando $T \rightarrow 0$, o espectro eletrônico do calor específico exibe um número infinito de oscilações de pequena amplitude simétricas e dispostas em torno da dimensionalidade fractal $d_{f}=\ln 2 / \ln 3=0,63092 \ldots$ Este comportamento é do tipo oscilatório em seu espectro de energia. Os espectros de energia de quasicristais do conjunto de Cantor triádico são absolutamente simétricos e uniformes. Por outro lado, vários espectros reais não são simétricos nem uniformemente espaçados. Desta forma, é necessária a utilização de um modelo que seja genérico e que possa tratar quaisquer tipos de espectros reais sem a preocupação com a simetria ou a quebra de simetria. O ponto de partida para a sustentação matemática deste modelo está ilustrado na Figura 4 abaixo.

Persp. Online: exatas \& eng., Campos dos Goytacazes, 19 (07) 20-35 - 2017 


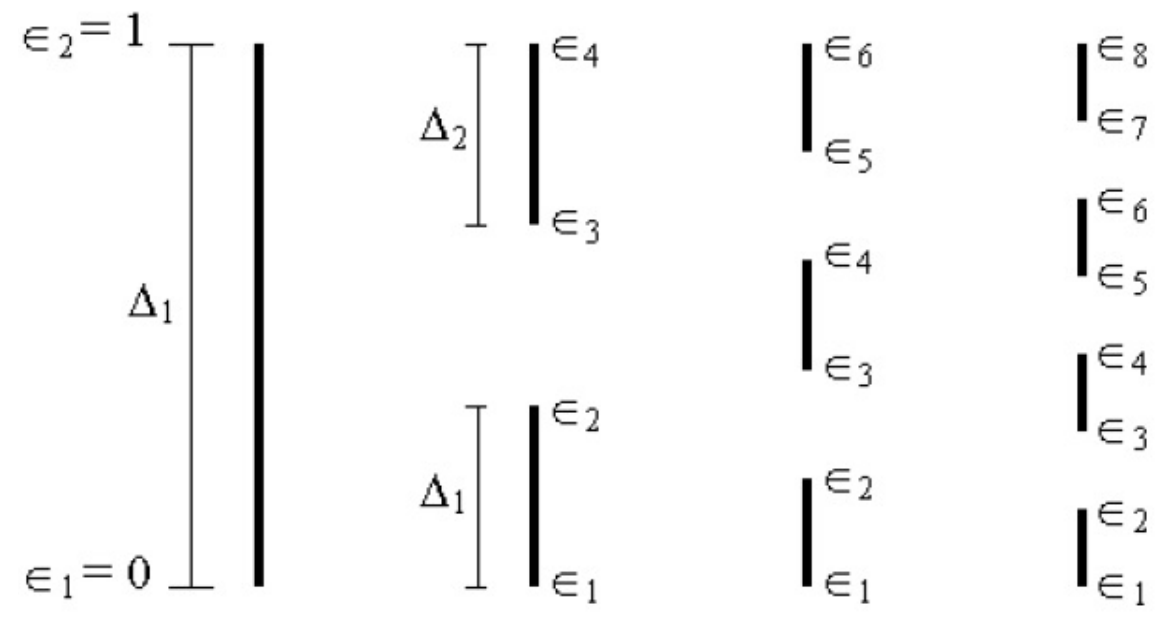

Figura 4 - Espectro de energia fractal genérico para um quasicristal.

essa figura é possível observar que:

$$
\begin{aligned}
& \Delta_{1}=\epsilon_{2}-\epsilon_{1} \Rightarrow \epsilon_{2}=\epsilon_{1}+\Delta_{1} \\
& \Delta_{2}=\epsilon_{4}-\epsilon_{3} \Rightarrow \epsilon_{4}=\epsilon_{3}+\Delta_{2}
\end{aligned}
$$

$$
\Delta_{i}=\epsilon_{2 i}-\epsilon_{2 i-1} \Rightarrow \epsilon_{2 i}=\epsilon_{2 i-1}+\Delta_{i}
$$

Assim, nesta hipótese, a representação do espectro da banda de energia; ou seja, o caso $n=1$ corresponde a um espectro contínuo de $\varepsilon_{1}$ a $\left(\varepsilon_{1}+\Delta_{1}\right)$. Para $n=2$, observa-se que no espectro da banda o primeiro ramo vai de $\varepsilon_{1}$ a $\left(\varepsilon_{1}+\Delta_{1}\right)$ e o segundo ramo vai de $\varepsilon_{3}$ a $\left(\varepsilon_{3}+\Delta_{2}\right)$ e assim, sucessivamente, para $n$ crescente. Tomando a densidade de nível no interior de cada banda como sendo constante, observa-se que ela é a mesma para todas as bandas numa dada hierarquia mesmo no caso fractal no limite $n \rightarrow \infty$. Este modelo é geral e pode ser aplicado a quaisquer espectros de energia de excitações eletrônicas em estruturas quasiperiódicas em quasicristais. Obviamente, o número de bandas depende da seqüência escolhida e da normalização no espectro de freqüência de tal modo que as bandas ficam dentro do limite 0 e 1 (em unidades de $\hbar w$ ). Ao se aplicar a Estatística Clássica de Maxwell-Boltzmann, a Função de Partição é dada por [18]: 
$Z_{n}=\int_{0}^{\infty} \rho(\varepsilon) e^{-\beta \varepsilon} d \varepsilon$

Onde $\beta=\frac{1}{T} Z_{N}=\int_{\epsilon_{1}}^{\epsilon_{2}} e^{-\beta \epsilon} d \in+\int_{\epsilon_{3}}^{\epsilon_{4}} e^{-\beta \epsilon} d \in+\cdots$ sendo $\mathrm{T}$ em na escala de Kelvin.

Foi considerada a constante de Boltzmann unitária; ou seja, $K_{B}=1$ e adotada a densidade de estados $\rho(\varepsilon)=1$. Na equação acima, $N$ é o número de bandas do espectro de energia do quasicristal.

Considerando $N$ bandas contínuas, a equação (10) é reescrita como se segue:

$$
Z_{N}=\int_{\epsilon_{1}}^{\epsilon_{2}} e^{-\beta \epsilon} d \in+\int_{\epsilon_{3}}^{\epsilon_{4}} e^{-\beta \epsilon} d \in+\cdots
$$

Essa equação pode ainda ser expressa como se segue:

$$
Z_{N}=\frac{1}{\beta} \sum_{i=1,3, \ldots}^{2 N-1}\left[\int_{\epsilon_{i}}^{\epsilon_{i+1}} e^{-\beta \epsilon} d \in\right]
$$

Considerando no sistema quasicristalino periódico de Fibonacci que $N=F_{n}$, após alguns cálculos e partindo da equação (12), obtém - se:

$$
Z_{N}=\frac{1}{\beta} \sum_{i=1,3, \ldots}^{2 N-1} e^{-\beta \epsilon_{i}}\left[1-e^{-\beta \Delta_{i}}\right]
$$

A partir do conhecimento da função de partição, é possível calcular o calor específico, C das estruturas quasicristalinas usando a seguinte equação:

$$
C_{N}=\beta^{2} \frac{\partial^{2} \ln Z_{N}}{\partial \beta^{2}}
$$


Tomando o logaritmo natural da equação (8) vem que:

$$
C_{N}=1+\frac{\beta f_{N}}{Z_{N}}-\frac{g_{N}^{2}}{Z_{N}^{2}}
$$

Onde,

$$
f_{N}=\sum_{i=1,3, \ldots}^{2 N-1}\left[\epsilon_{i}^{2} e^{-\beta \epsilon_{i}}-\epsilon_{i+1}^{2} e^{-\beta \epsilon_{i+1}}\right]
$$

Sendo a função $g_{n}$ relacionada ao calor específico dado por:

$$
g_{N}=\sum_{i=1,3, \ldots}^{2 N-1}\left[\epsilon_{i} e^{-\beta \epsilon_{i}}-\epsilon_{i+1} e^{-\beta \epsilon_{i+1}}\right]
$$

Desta forma, é possível determinar o calor específico das estruturas quasicristalinas relacionando sempre aos efeitos eletrônicos em quasicristais.

\section{RESULTADO E DISCUSSÃO}

O conjunto fractal discreto do calor específico em estruturas quasiperiódicas, descreve polaritons de plasmon na superfície relacionado à super-rede quasiperiódicas de Fibonacci para o calor específico no quasicristal, este fenômeno é do tipo um espectro fractal emerge quando o número de geração $n$ que vai para infinito. Na representação do espectro de frequência (ou de energia) é fácil ver que para $n=6$ existem dois níveis discretos e sucessivamente, gerando uma de geração de Fibonacci. O calor específico de deste sistema, é uma função da temperatura, conforme mostrada na Figura 5, nela podemos ver a existência de um pico que aumenta, para todos os números de geração mostrados aqui, num intervalo entre $1.10^{-2}$ a $6 \cdot 10^{-2}$. Também é fácil verificar os dois limites bem definidos, $T^{-2},(T \rightarrow \infty)$ quando $T \rightarrow 0$ e $C_{N}$ vai à zero em $T^{-2},(T \rightarrow \infty)$ este gráfico é do tipo log-log do 
calor específico versus a temperatura, que vem confirmar oscilações em regiões de baixas temperaturas.

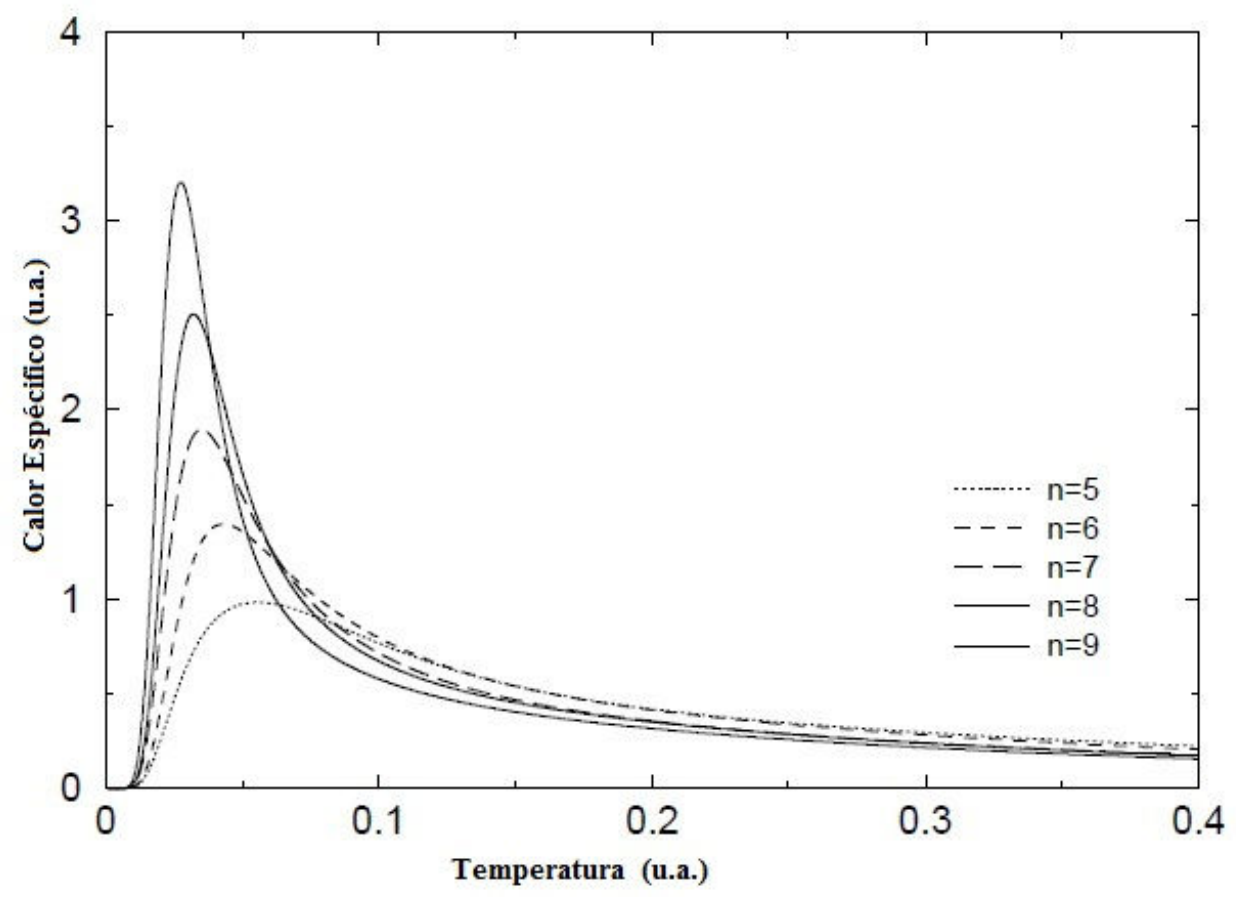

Figura 5 - O gráfico do calor específico em estrutura quasiperiódica de Fibonacci.

\section{CONCLUSÕES}

* O estudo sobre ligas quasicristalinas do tipo $\mathrm{Al} \mathrm{Cu} F$ foi fundamentado nas propriedades estruturais e eletrônicas e considerou que o sistema quasiperiódico obedece à regra matemática de Fibonacci (sequência generalizada);

O espectro de frequência (ou de energia) que para $n=6$ apresenta dois níveis discretos e sucessivamente, gerando uma de geração de Fibonacci. O calor específico deste sistema é uma função da temperatura, pois podemos ver um pico que aumenta, para todos os números de geração mostrados aqui, num intervalo entre $1.10^{-2}$ a $6.10^{-2}$, nestes limites é fácil que em $T^{-2},(T \rightarrow \infty)$ quando $T \rightarrow 0$ e $C_{N}$ vai à zero em $T^{-2},(T \rightarrow \infty)$, onde confirmarmos oscilações em regiões de baixas temperaturas ao seu espectro de energia, que sofre oscilações discretas, do tipo fractal;

* Estudamos as propriedades do calor específico do espectro de energia dos quasicristais obtidos de estruturas quasiperiódicas via sequência Fibonacci generalizada; 
* Observamos a existência de uma similaridade no padrão do comportamento oscilatório do seu calor específico usando o formalismo da mecânica estatística entre os números de sequência de Fibonacci generalizada (tipo fractal) e o conjunto de Cantor triádico;

* A análise algébrica dos espectros de energia nas estruturas quasicristalinas mostra uma forte desordenação e se constitui em uma ferramenta adequada à determinação das propriedades eletrônicas e do calor específico a nível quântico.

\section{AGRADECIMENTOS}

Os autores agradecem ao CNPq pelo apoio financeiro

\section{REFERÊNCIAS}

[1]. PINTO, M. Z. ; PILLACA, M. LANDAURO, C. V. Estudio de la influencia de la temperatura y el tiempo de tratamiento térmico en la estabilidad del cuasicristal $\mathrm{Al}_{64} \mathrm{Cu}_{23} \mathrm{Fe}_{13}$. Revista de Investigación de Física 15, 121501101. pp.1 -6,2012.

[2]. BARUA, P.; MURTY, B. S.; SRINIVAS, V. Mechanical alloying of Al-Cu-Fe elemental powders. Materials Science and Engineering A, 304-306 . pp. 863-866, 2001.

[3]. ROY, M. Formation and magnetic properties of mechanically alloyed $\mathrm{Al}_{65} \mathrm{Cu}_{20} \mathrm{Fe}_{15}$ quasicrystal. Journal of Magnetism and Magnetic Materials 302. pp.52-55,2006.

[4].SRIVASTAVA,V.C.;HUTTUNEN-SAARIVIRTA,E.;CUI,C.;UHLENWINKEL,V.; SCHULZ,A.;MUKHOPADHYAY,N.K. Bulk synthesis by spray forming of $\mathrm{Al}-\mathrm{Cu}-\mathrm{Fe}$ and $\mathrm{Al}-\mathrm{Cu}-$ Fe-Sn alloys containing a quasicrystalline phase. Journal of Alloys and Compounds 597, pp.258$268,2014$.

[5]. AgOStinHO, L.C.L. Estudo da Aplicabilidade dos Quasicristais AlCuFe em Reações Catalíticas na Oxidação do Metanol, Dissertação (Mestrado em Ciências de Materiais),Universidade Federal da Paraíba,João Pessoa-Paraíba,2009.

[6]. SVIRIDOVA, T.A. SHEVCHUKOV, A.P. SHELEKHOV, E.V.; DIAKONOV, D.L.; GORSHENKOV, M.V. KALOSHKIN, S.D. TCHERDYNTSE, V.V. LI, Z.; FAN,G. Nano

Persp. Online: exatas \& eng., Campos dos Goytacazes, 19 (07) 20-35 - 2017 seer.perspectivasonline.com.br 
quasicrystalline Phase in Mechanically Alloyed and Heat-Treated $\mathrm{Al}_{73} \mathrm{Cu}_{11} \mathrm{Cr}_{16}$. Acta Physica Polonica A 126, pp.599-602, 2014.

[7]. LOGRASSO, T. A.; DELANEY, D. W. Preparation of large single grains of the quasicrystalline icosahedral Al-Cu-Fe $\psi$ phase. Journal of Materials Research 11. pp. 2125- 2127,1996.

[8]. BERGER, C. in: Lectures on Quasicrystals, Eds.F. Hippert, D. Gratias, Les Editions de Physique, Les Ulis, p. 463, 1994.

[9]. SCHETMAN, D.; BLECH, I.; GRATIAS, D.; CANHN, J.W. Metallic phase with long-range orientational order and no translational symmetry. Phys.Rev.Lett.; 53. pp.1951-4, 1984.

[10]. AGOSTINHO, L. C. L.; BARBOSA, C.M.B.M.; NASCIMENTO, L.; RODBARI,J.R. Catalytic Dehydration of Methanol to Dimethyl Ether (DME) Using The $\mathrm{Al}_{62,2} \mathrm{Cu}_{25,3} \mathrm{Fe}_{12,5}$ Quasicrystalline Alloy. Journal Chem. Eng. Process. Technol. 4,pp.2-8,2013.

[11]. POON, S.J. Electronics Properties of Quasicrystals: An Experimental Review, Adv.Phys.41. p.303, 1993.

[12]. BONASSO, N.; PIGEAT, P. Preparation of Al-Cu-Fe ultra-thin quasicrystalline films without protective coatings by MBE: influence of processing. Materials Science and Engineering A 349, pp.224-229, 2003.

[13]. MANDEL, Even-Dar,S.; LIFSHITZ,R. Electronic energy spectra of square and cubic Fibonacci quasicrystals. Philosophical Magazine 88:13-15, 1-21.pp. 2261-2273, 2008.

[14]. Jamshidi, L.C.L.A . Desidratação Catalítica do Metanol para Dimetil Éter Utilizando Liga Quasicristalina e Zeolita Nanoestruturada, Projeto de Tese de Doutorado (Engenharia Química), Universidade Federal de Pernambuco, Recife- Pernambuco.pp.1-46,2013.

[15]. CHAIKIN, P.M.; LUBENSKY, T.C. Principles of condensed matter physics, Cambridge University Press, Cambridge, 1995.

[16]. BHELIN, H. Electronic distributions in quasicrystalline AlCuFe alloys. J.Phys.Cond.Matter 6; $8772-17,1994$

[17]. SADOC,A. ;BELIN,E. ;DANKHAZIC,Z. ; FLANK,A.M. Evidence of a wide pseudo-gap in AlCuFe icosahedral alloys. Journal of Non-Crystalline Solids 153-154:2,pp. 338-342,1992.

Persp. Online: exatas \& eng., Campos dos Goytacazes, 19 (07) 20-35 - 2017 seer.perspectivasonline.com.br 
[18]. NASCIMENTO. L. Propriedades Eletrônicas e Modelos em Quasicristais, Dissertação (Mestrado em Ciências de Materiais), Universidade Federal da Paraíba, João Pessoa-Paraíba, 2009.

Persp. Online: exatas \& eng., Campos dos Goytacazes, 19 (07) 20-35 - 2017 seer.perspectivasonline.com.br 